\title{
Mice lacking mitochondrial ferritin are more sensitive to doxorubicin-mediated cardiotoxicity
}

\author{
Federica Maccarinelli • Elena Gammella • Michela Asperti • Maria Regoni • \\ Giorgio Biasiotto • Emilia Turco • Fiorella Altruda • Silvia Lonardi • Laura Cornaghi • \\ Elena Donetti • Stefania Recalcati • Maura Poli • Dario Finazzi • Paolo Arosio • \\ Gaetano Cairo
}

Received: 6 September 2013 /Revised: 9 February 2014 / Accepted: 18 March 2014 /Published online: 13 April 2014

(C) The Author(s) 2014. This article is published with open access at Springerlink.com

\begin{abstract}
Mitochondrial ferritin is a functional ferritin that localizes in the mitochondria. It is expressed in the testis, heart, brain, and cells with active respiratory activity. Its overexpression in cultured cells protected against oxidative damage and reduced cytosolic iron availability. However, no overt phenotype was described in mice with inactivation of the FtMt gene. Here, we used the doxorubicin model of cardiac injury in a novel strain of FtMt-null mice to investigate the antioxidant role of FtMt. These mice did not show any evident phenotype, but after acute treatment to doxorubicin, they showed enhanced mortality and altered heart morphology with fibril disorganization and severe mitochondrial damage. Signs of mitochondrial damage were present also in mock-treated $\mathrm{FtMt}^{-/-}$mice. The hearts of saline- and doxorubicin-treated $\mathrm{FtMt}^{-1}$ mice had higher thiobarbituric acid reactive substance levels, heme oxygenase 1 expression, and protein oxidation, but did not differ from $\mathrm{FtMt}^{+/+}$in the cardiac damage marker B-type natriuretic peptide (BNP), ATP levels, and apoptosis. However, the autophagy marker LC3 was activated. The results show
\end{abstract}

Electronic supplementary material The online version of this article (doi:10.1007/s00109-014-1147-0) contains supplementary material, which is available to authorized users.

F. Maccarinelli $\cdot$ M. Asperti $\cdot$ M. Regoni $\cdot$ G. Biasiotto $\cdot$ S. Lonardi $\cdot$ M. Poli $\cdot$ D. Finazzi $\cdot$ P. Arosio $(\square)$

Department of Molecular and Translational Medicine, University of Brescia, Viale Europa 11, 25123 Brescia, Italy

e-mail: arosio@med.unibs.it

E. Gammella $\cdot$ L. Cornaghi $\cdot$ E. Donetti $\cdot$ S. Recalcati $\cdot$ G. Cairo Department of Biomedical Sciences for Health, University of Milano, Via Mangiagalli 31, Milan, Italy

E. Turco $\cdot$ F. Altruda

Department of Molecular Biotechnology and Health Sciences,

University of Torino, Via Nizza, 10126 Torino, Italy that the absence of FtMt, which is highly expressed in the heart, increases the sensitivity of heart mitochondria to the toxicity of doxorubicin. This study represents the first in vivo evidence of the antioxidant role of FtMt.

\section{Key message}

- Mitochondrial ferritin (FtMt) expressed in the heart has a protective antioxidant role.

- Acute treatment with doxorubicin caused the death of all $\mathrm{FtMt}^{-/-}$and only of $60 \% \mathrm{FtMt}^{+/+}$mice.

- The hearts of $\mathrm{FtMt}^{-/-}$mice showed fibril disorganization and mitochondrial damage.

- Markers of oxidative damage and autophagy were increased in $\mathrm{FtMt}^{-/-}$hearts.

- This is the first in vivo evidence of the antioxidant role of FtMt.

Keywords Ferritin · Oxidative damage $\cdot$ Mitochondria Doxorubicin

\section{Introduction}

Mitochondrial ferritin (FtMt) is a recently identified ferritin type that accumulates specifically in the mitochondria [1]. Human FtMt, which is encoded by an intronless gene, is synthesized as a precursor with a long N-terminal targeting sequence that is cleaved in the mature protein. The mature FtMt has a functional ferroxidase center and forms stable ferritin shells that readily accumulate iron [2]. Its 3D structure is analogous to that of the H-ferritin [3]. The FtMt transcript does not contain a functional iron responsive element (IRE) sequence, and thus, FtMt expression is not controlled by intracellular iron levels. In humans, FtMt was found to be expressed in the testis [4], neurons [5], and in the erythroblasts 
of subjects with genetic or acquired sideroblastic anemia [6], where it is responsible of the mitochondrial iron deposits of the sideroblasts. Since human FtMt is not detectable in cultured cells, most of the data on its function have been obtained by analyzing the phenotype of overexpressing cells. These studies showed that, by virtue of its ferroxidase activity, FtMt actively sequesters iron inside the mitochondria at the expense of cytosolic iron [7]. It also reduces iron-mediated oxidative damage of mitochondria [8] and rescues some defects caused by frataxin deficiency in yeast [9] and HeLa cells [10]. More recently, it has been shown that overexpression of FtMt in K562 erythroid cells reduced Jak/STAT signaling and increased apoptosis [11]. In sideroblasts, erythroid progenitors FtMt expression occurred at the early stage of cell differentiation and was accompanied by reduced iron availability and increased apoptosis [12, 13]. Moreover, FtMt expressing cells transplanted in nude mice grew more slowly than their control counterpart [14]. Altogether, FtMt seems to protect mitochondria against iron-dependent oxidative damage and also modifies cellular iron distribution by attracting iron from the cytosol to mitochondria [15]. Specific antibodies have been raised against mouse FtMt that were used to study its distribution in mouse organs. It was found that FtMt is strongly expressed in the testis, particularly in the spermatocytes, and also in the heart, kidney, Purkinje cells, and some neurons, and generally in cells with high respiratory activity that actively use iron enzymes and produce reactive oxygen species (ROS) [16]. In line with the results obtained in the cell lines, FtMt was also found to decrease the sensitivity of mitochondria to oxidative damage in SLA mice [17] and to protect neuronal cells from oxidative damage [18-20]. The role of FtMt in neurodegenerative diseases has been recently reviewed [21]. However, the recently described FtMt-deficient mice in the C57BL/6J strain did not show any evident phenotype and produced siderocytes/sideroblasts similar to the control $\mathrm{FtMt}^{+/+}$even under conditions of vitamin B6 deficiency [22]. It is unlikely that the lack of phenotype in FtMt deficiency is due to redundancy of the function, since FtMt is the only known mitochondrial iron storage protein. Although FtMt appears dispensable under physiological conditions, it may protect against damages under specific conditions. FtMt is highly expressed in the heart; thus, we considered that it may protect its mitochondria against oxidative damage, in particular from the injury induced by doxorubicin (Dox), a well-characterized anthracycline whose extensive use for the cure of a variety of tumors is hampered by a recognized cardiotoxicity [23-25]. Anthracyclines, including Dox, possess a high affinity for cardiolipin, a negatively charged phospholipid of the inner mitochondrial membrane [26], and hence, they are retained at high concentrations in the mitochondrial compartment. Confocal microscopy experiments showed that in H9c2 cardiomyocytes, Dox localized to mitochondrial sites of redox cycling and ROS formation [27].
Therefore, mitochondria have been repeatedly suggested as the most important target for anthracyclines cardiotoxicity [24]. In this context, the availability of intracellular reactive iron, which catalyzes ROS formation, appears to be determinant for Dox cardiotoxicity, as shown by the protective effect of iron chelation in patients and in experimental models [28], whereas iron overload have been shown to exacerbate the cardiotoxic effects of the drug [29-32]. In particular, mitochondrial iron may play a significant role in leading to elevated mitochondrial ROS formation, and it has been recently shown that overexpression of FtMt can protect HeLa cells from Dox toxicity [33] and that Dox treatment strongly induces FtMt expression in neonatal rat cardiomyocytes [34]. Moreover, a recent study showed that Dox cardiotoxicity is mediated by mitochondrial iron accumulation and that heartspecific deletion of mitochondrial iron exporter ABCB8 increased mice sensitivity to Dox cardiotoxicity, whereas its overexpression was protective [34].

In this study, we tested the hypothesis that the heart of FtMt-deficient animals might be more sensitive to cardiotoxic drugs; with this aim, we used a model of acute Dox cardiotoxicity in a novel strain of FtMt-null mice to evaluate the role of FtMt in heart protection from anthracyclinedependent oxidative injury.

\section{Materials and methods}

Animals All the procedures followed animal protection laws and institutional guidelines of the European Convention for the Protection of Laboratory Animals. The study was approved by the Institutional Animal Care and Use Committee of the University of Brescia and the Italian Ministry of Science and Research.

Gene targeting construct A 5,030-bp cassette that included the Lac-Z gene followed by the hybrid pGK-EM7 promoter and the Neo gene was cloned into the pBluescript-SK (pBSK) plasmid. The cassette was flanked by a $5^{\prime}$ homology arm of 275 bp upstream the FtMt start codon and a short 3' arm of 270 bp downstream the FtMt stop codon. The construct named pBSK +5-b-n-3 was verified by DNA sequencing and then used together with the vector $\mathrm{pBSK}+\mathrm{moMtF}$ of $21.5 \mathrm{kbp}$ containing the full mouse gene and flanking sequences in the recombineering system [35] to obtain the gene targeting vector that had homology arms at $5^{\prime}$ and $3^{\prime}$ of about $8 \mathrm{kbp}$. The construct was then used for electroporation of mouse ES cells, which were then selected in G418 medium, and one clone was microinjected in embryos. The born mice showed about $50 \%$ chimerism and were crossed with C57BL/6J mice, and the newborn genotyped by PCR as described below. Mice were bred in mixed C57BL/6J x 129 genetic background. 
Genotyping The mice were genotyped by PCR using a common Fwd-primer upstream the $5^{\prime}$-arm and two different Revprimers, one specific for the mouse mitochondrial ferritin (moFtMt) gene (see Table 1), using the following PCR conditions: 5 min at $95^{\circ} \mathrm{C}, 5$ cycles $\left(30 \mathrm{~s}\right.$ at $94^{\circ} \mathrm{C}, 30 \mathrm{~s}$ at $50^{\circ} \mathrm{C}$, $45 \mathrm{~s}$ at $\left.72{ }^{\circ} \mathrm{C}\right), 30$ cycles $\left(30 \mathrm{~s}\right.$ at $94{ }^{\circ} \mathrm{C}, 30 \mathrm{~s}$ at $55^{\circ} \mathrm{C}, 45 \mathrm{~s}$ at $72^{\circ} \mathrm{C}$ ), followed by $10 \mathrm{~min}$ at $72^{\circ} \mathrm{C}$.

DOX treatments To induce cardiotoxicity in the survival studies, 84-day-old female mice were injected intraperitoneally with saline or a single dose (15 mg/kg of body weight) of Dox (Sigma, Milan, Italy) and then, the mice were followed for 30 days. In other experiments, female mice were treated as above and sacrificed after 4 days for the analysis of the heart, or male mice were sacrificed after 30 days for analysis of the testis. The organs were harvested, weighted, frozen in liquid nitrogen, and stored at $-80{ }^{\circ} \mathrm{C}$.

Quantitative real-time polymerase chain reaction ( $q R T$ $P C R)$ Total heart or testis RNA purified using TRI reagent ${ }^{\circledR}$ (Sigma) was reverse transcribed into cDNA with Proto Script M-MuLV First Strand cDNA Synthesis Kit (New England Biolabs, Italy), and the obtained cDNA served as a template for real-time PCR, based on the TaqMan methodology (Life Technologies). Primers (Applied Biosystems) and parameters are described in detail in Table 1. For evaluation of beta-

Table 1 Primers and Taqman assays used

\begin{tabular}{ll}
\hline Genotyping primers & \\
B5-Fwd & 5'-ACGCGTCGACCTTGTGTTAGTAATTC \\
& AGCC-3' \\
mT4-Rev & 5'-CAGAGTATGTAAGTCCAGCAGC-3' \\
LacZ-Rev & 5'-GGGACGACGACAGTATCGGCCT-3' \\
RT-PCR primers & \\
BGAL-FwdNew & 5'-GCACGGTTACGATGCGCCCA-3' \\
BGAL-RevnNew & 5'-GCGCTGGAGTGACGGCAGTT -3' \\
mT1-For & 5'-TATTTCCTTCGCCAGTCCCTG-3' \\
mT4R-Rev & 5'-CAGAGTATGTAAGTCCAGCAGC-3' \\
mHPRT1-For & 5'-GCTTGCTGGTGAAAAGGACCTCTC \\
mHPRT1-Rev & GAAG-3' \\
5'-CCCTGAAGTACTCATTATAGTCAAGG \\
TaqMan gene & GCAT-3' \\
HO-1 & Assay ID \\
BNP & Mm00516005_m1 \\
p53 & Mm01255770_g1 \\
Rn18s & Mm00519571_m1 \\
\hline
\end{tabular}

The genotyping primers were used to verify the presence of FtMt gene or of LacZ gene in the FtMt locus. RT-PCR primers were used for identification of the transcripts of FtMt, $\beta-G a l$, and HPRT1 in the testis of the mice. The TaqMan gene expression assays were used to quantify HO-1, $\mathrm{BNP}, \mathrm{p} 53$, and Rn18s transcripts in the heart of the treated mice galactosidase ( $\beta$-gal) and FtMt transcripts, we used RT-PCR with the primers described in Table 1 and cycling conditions as follows: $5 \mathrm{~min}$ at $95^{\circ} \mathrm{C}, 30$ cycles $\left(30 \mathrm{~s}\right.$ at $94{ }^{\circ} \mathrm{C}, 30 \mathrm{~s}$ at $60{ }^{\circ} \mathrm{C}$, and $30 \mathrm{~s}$ at $72{ }^{\circ} \mathrm{C}$ ), followed by $10 \mathrm{~min}$ at $72{ }^{\circ} \mathrm{C}$.

Western blotting Tissue lysates were prepared in RIPA buffer, incubated on ice for $30 \mathrm{~min}$, and centrifuged at 13,000 rpm for $10 \mathrm{~min}$. Proteins in supernatant were separated on nondenaturing or SDS-PAGE and transferred onto nitrocellulose membranes (GE Healthcare, Milan, Italy). Membranes were processed and incubated with primary antibodies against mouse FtMt, $\mathrm{H}$ and L ferritins [16], $\beta$-gal, LC3 and GAPDH (Sigma), and horseradish peroxidase (HRP)-conjugated secondary antibodies. The antigens were detected using an immunodetection kit (ECL Basic, Amersham Biosciences). For evaluation of ferritin iron, the non-denaturing gels were stained with Prussian blue and then the color enhanced using diaminobenzidine (DAB) and $\mathrm{H}_{2} \mathrm{O}_{2}$ (Ft-iron). For visualization and densitometry, we used the Kodak Image Station 440CF (Kodak).

Determination of ATP content Heart tissue samples (10 mg) were homogenized in perchloric acid, and ATP was measured by a colorimetric assay using a commercial kit (ATP Colorimetric assay kit; Biovision, Italy) following manufacturer's instructions.

Immunohistochemistry Mouse testes were dissected according to approved protocols. Mice were transcardially perfused with saline buffer, and the organs were removed and stored in $4 \%$ formaldehyde for 2 days and then paraffin embedded. Four-micrometer sections of $\mathrm{FtMt}^{+/+}$and $\mathrm{FtMt}^{-/-}$mouse testes were deparaffinized in xylene and rehydrated through a series of alcohol gradients. Sections underwent antigen retrieval with $0.05 \%$ protease type XIV digestion for $5 \mathrm{~min}$ at $37{ }^{\circ} \mathrm{C}$, and endogenous peroxidase activity was quenched by $0.3 \% \mathrm{H}_{2} \mathrm{O}_{2}$ in methanol for $20 \mathrm{~min}$. The tissues were incubated for $1 \mathrm{~h}$ at room temperature with rabbit anti-mouse FtMt antiserum [16] diluted 1:1,000 and then with Rabbit-on-Rodent HRP-Polymer (BIOCARE Medical, CA, USA) for $30 \mathrm{~min}$. Sections were then incubated for $5 \mathrm{~min}$ with 3,3'-diaminobenzidine (DAB), washed, counterstained with hematoxylin, dehydrated, and coverslipped.

Light and electron microscopy Small blocks of heart were fixed in $3 \%$ glutaraldehyde (Acros Organics, Thermo Fisher Scientific, Waltham, MA, USA) in Sorensen phosphate buffer $\left(0.1 \mathrm{M}\right.$; pH 7.4) overnight at $4{ }^{\circ} \mathrm{C}$, post-fixed with $1 \%$ osmium tetroxide in $0.1 \mathrm{M}$ Sorensen phosphate buffer for $30 \mathrm{~min}$, dehydrated, and embedded in Araldite (Fluka-Sigma Aldrich). Semi-thin sections, 2- $\mu \mathrm{m}$ thick, were stained with toluidine blue. For electron microscopy, ultrathin sections 
$(200 \mathrm{~nm})$ were obtained with an Ultracut ultramicrotome (Reichert-Jung, Leica, Microsystems GmbH, Wetzlar, Germany), stained with uranyl acetate and lead citrate, and observed with a JEM 1010 transmission electron microscope (Jeol, Tokyo, Japan).

TBARS assay Lipid peroxidation was assessed using the thiobarbituric acid reactive substance (TBARS) assay. Heart tissue was homogenized in $150 \mathrm{mM} \mathrm{KCl}$, and an aliquot was used for the determination of protein concentration. A $100-\mu \mathrm{l}$ aliquot of tissue homogenate in triplicate was mixed with $0.5 \mathrm{ml}$ of $0.22 \mathrm{M}$ butylated hydroxytoluene (Sigma), $3 \mathrm{ml}$ of $1 \%$ phosphoric acid, and $1 \mathrm{ml}$ of $0.6 \%$ thiobarbituric acid (Sigma). Samples were incubated at $100{ }^{\circ} \mathrm{C}$ for $60 \mathrm{~min}$ and then cooled at room temperature. Lipids were extracted using $5 \mathrm{ml}$ of isopropanol:chloroform $(11: 7, v / v)$, centrifuged at $2,000 \times \mathrm{g}$ for $10 \mathrm{~min}$, and the absorbance of the upper layer was read at $535 \mathrm{~nm}$. The amount of TBARS was quantified using a standard curve of malonaldehyde bis (dimethyl acetal), (MDA, Sigma).

Caspase activity assay Caspase-3 activity was determined using the ApoTarget Caspase Colorimetric Assay kit (Invitrogen, Monza, Italy), following the manufacturer's protocol. In brief, tissue samples were lysed in $100 \mu \mathrm{l}$ of lysis buffer and protein concentrations in samples determined using the Bio-Rad protein assay. After incubation on ice for $10 \mathrm{~min}$, the samples were centrifuged at $16.000 \times g$ for $3 \mathrm{~min}$ at $4{ }^{\circ} \mathrm{C}$. Each supernatant was mixed with $50 \mu \mathrm{l}$ of $2 \mathrm{X}$ reaction buffer/DTT mix and $5 \mu$ of $1 \mathrm{mM}$ caspase-3 substrate (DEVD-pNA, $50 \mu \mathrm{M}$ final concentration), and then, the samples were incubated for $2 \mathrm{~h}$ at $37{ }^{\circ} \mathrm{C}$ in the dark. Developed color was measured at $405 \mathrm{~nm}$, and caspase activity was calculated in terms of absorbance units per microgram protein.

Oxidized protein detection Hearts were homogenized in lysis buffer (Tris $\mathrm{HCl} 20 \mathrm{mM}, \mathrm{pH}$ 7.4, 0.1 \% SDS, and protease inhibitor). After centrifugation at $13.000 \mathrm{rpm}$ for $10 \mathrm{~min}$ at $4{ }^{\circ} \mathrm{C}$, the supernatant was added with $50 \mathrm{mM} \mathrm{DTT}$, frozen, and stored at $-20{ }^{\circ} \mathrm{C}$. Oxidized proteins in protein extracts were detected using the OxyBlot Protein Oxidation Detection Kit (Millipore) following manufacturer's instructions. In brief, the samples were reacted with 2,4-dinitrophenylhydrazine (DNPH) for derivatization to 2,4-dinitrophenylhydrazone (DNP), then were loaded on SDS-PAGE, blotted, and incubated with an anti-DNP antibody. The bound activity was revealed by ECL (GE Healthcare).

Statistical analysis Differences were analyzed using a Student's $t$ test for paired samples, and comparisons were made using appropriate analysis of variance (ANOVA). The significance level was set to $p<0.05$.

\section{Results}

Production of the $\mathrm{FtMt}^{-/-}$mice

The structure of the gene targeting construct is shown in Fig. 1a. The intronless FtMt gene of 714 bp was replaced by a 5,030-bp cassette that contained lac-Z gene under the control of the FtMt promoter and the gene for neomycin resistance under the control of the strong pGK promoter. Genotyping was done by PCR using a common Fwd primer and specific Rev primers for wild type (wt) or recombinant gene (Fig. 1). This allowed to distinguish the $\mathrm{FtMt}^{+/+}$, from $\mathrm{FtMt}^{+/-}$and $\mathrm{FtMt}^{-/-}$strains (Fig. 1b). To verify the absence of FtMt expression, we initially analyzed the testis, where its level is the highest [16]. RT-PCR analyses showed the absence of FtMt transcript and the presence of $\beta$-gal transcript encoded by the Lac-Z gene in the $\mathrm{FtMt}^{-/-}$mice. The FtMt, but not the $\beta$-gal transcript, was present in $\mathrm{FtMt}^{+/+}$, while $\mathrm{FtMt}^{+/-}$ displayed both transcripts, as expected (Fig. 1c); possibly because of the low level of expression of FtMt, or of modification of the methylation status, we could not observe any $\beta$ gal activity in the testis of the $\mathrm{FtMt}^{-/-}$mice or in any other tissue (results not shown). Western blotting of testis homogenates detected a FtMt band in the $\mathrm{FtMt}^{+/+}$mice, but not in the $\mathrm{FtMt}^{-/-}$mice, and a $\beta$-gal band in $\mathrm{FtMt}^{-/-}$, but not in $\mathrm{FtMt}^{+/+}$ mice (Fig. 1d). It confirmed also the absence of FtMt in the kidney and, more important, in the cardiac tissue of $\mathrm{FtMt}^{-/}$ mice (Fig. 1e). As a further control, we performed immunohistochemical staining of slices from the testis. A stain was evident in the seminiferous tubule of the $\mathrm{FtMt}^{+/+}$mice that corresponded to the spermatocytes, whereas no staining was observed in the testis of $\mathrm{FtMt}^{-/-}$mice. (Fig. 1f, g). This also confirmed the specificity of the antibody.

\section{$\mathrm{FtMt}^{-/-}$phenotype}

The $\mathrm{FtM}^{+/-}$and $\mathrm{FtMt}^{-/-}$mice did not show any evident phenotype. They consumed the expected amount of food and behaved normally, also after 16 months of age. We did not observe any overt defect in fertility, both in males and females $\mathrm{FtMt}^{-/-}$. This corresponds to the description of $\mathrm{FtMt}^{-/-}$mice in the C57B/6J strain [22]. FtMt is known to be preferentially expressed in the testis, heart, kidney, and some neurons [16]. Being FtMt highly expressed in the heart, we suspected that the heart of FtMt-deficient animals may be more sensitive to cardiotoxic drugs. To test this hypothesis, we treated the mice with Dox, which is a well-known cardiotoxic drug [23-25]. In our experimental protocol, we used the same dose and timepoint recently used by us to investigate acute Dox cardiotoxicity [36] and we treated 12-week-old (84-day) female mice with a single intraperitoneal injection of $15 \mathrm{mg} / \mathrm{kg}$ to induce acute toxicity. The Kaplan-Meier survival plot reported in Fig. 2 shows that upon Dox challenge, the mortality 
Fig. 1 Construction and genotyping of $\mathrm{FtMt}^{-/-}$mice. a Schematic of the FtMt locus and targeting vector, the arrows indicate the PCR primers for genotyping. b Genotyping gel of mice wild type $(+/+)$,

heterozygous $(+/-)$, and homozygous $(-/-)$ for the inactivated FtMt allele. In the M lane, a DNA molecular weight ladder was loaded. The size of the expected PCR amplicons is indicated. $\mathbf{c}$ RT-PCR analysis of testis RNA extracted from wild type $(+/+)$, heterozygous $(+/-)$, and homozygous $(-/-)$ mice and amplified with primers for FtMt, beta-galactosidase ( $\beta$-gal, encoded by the Lac-Z gene), and the housekeeping transcript HPRT1. d Immunoblotting of protein extracts from the testis of wild type $(+/+)$, heterozygous $(+/$ $-)$, and homozygous (-l-) mice overlaid with antibodies for mouse FtMt, $\beta$-gal, and GAPDH as loading calibrator. $\mathbf{e}$ Immunoblotting of protein extracts from various tissues of wild type $(+/+)$ and homozygous $(-/-)$ mice, overlaid with antibodies for mouse FtMt. (f,g) Immunohistochemical detection of FtMt in the testis of wild type (f) and FtMt(-/-) (g) mice. Bar $50 \mu \mathrm{m}$
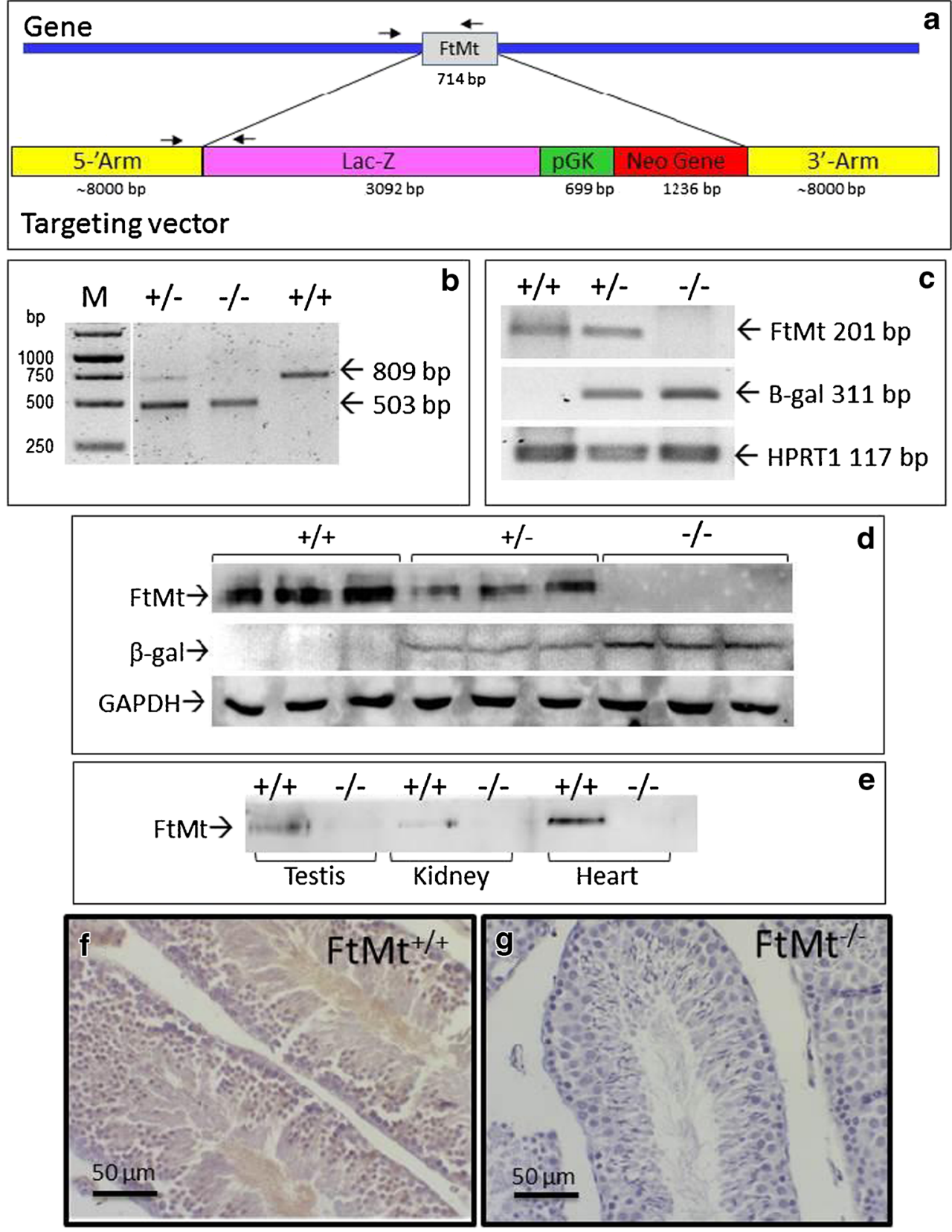

of $\mathrm{FtMt}^{-1-}$ mice was much higher than that of wild type animals. None of the mice without FtMt survived more than 6 days, whereas one third of $\mathrm{FtMt}^{+/+}$animals was still alive more than 3 weeks after treatment.

To further evaluate the role of FtMt in Dox-dependent cardiotoxicity, wild type and $\mathrm{FtMt}^{-/-}$female mice were treated with saline or Dox $(15 \mathrm{mg} / \mathrm{kg})$ and sacrificed 4 days later; no mortality was observed, in line with the findings of our previous study [36]. Transmission electron microscopy analysis did not show evident differences between the hearts of control wild-type mice before and after Dox treatment (Fig. 3a, b). Both the fibril organization and the mitochondrial morphological features were comparable in these two groups. Also, the histopathogical hallmarks of anthracycline cardiotoxicity were absent in the treated $\mathrm{FtMt}^{+/+}$group [37]. In contrast, the hearts of $\mathrm{FtMt}^{-/}$mice showed some defects: in the untreated ones, scattered mitochondria had incomplete cristae (Fig. 3c, arrowheads) although fibrils were regularly arranged (Fig. 3e). After Dox treatment, the damage was more severe with condensation and fragmentation of most myofibrils in some fields (arrows in Fig. 3f). Also, mitochondrial damage was more evident with zones in which the cristae were absent (Fig. 3d, arrowheads) and others with cristae completely disrupted (Fig. 3f). This type of morphology was observed in all the three animals analyzed in TEM. In no sample we could detect evident morphological signs suggesting apoptosis, e.g., 


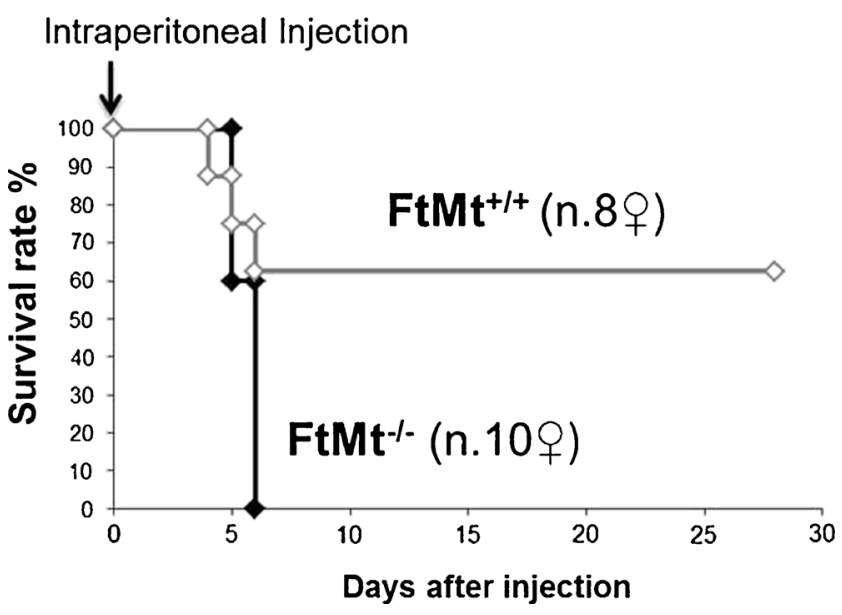

Fig. 2 Cumulative survival in mice treated with Dox. Kaplan-Meier plot of animal survival. Twelve-week-old female mice $\left(8 \mathrm{FtMt}^{+/+}\right.$and 10 $\mathrm{FtMt}^{-/}$) were subjected to a single intraperitoneal injection of $15 \mathrm{mg} / \mathrm{kg}$ Dox

chromatin condensation, nuclear fragmentation, and apoptotic bodies. Observation at the light microscopic level of toluidine blue-stained heart semi-thin sections of $\mathrm{FtMt}^{+/+}$mice exposed to Dox or saline, and untreated $\mathrm{FtMt}^{-/-}$mice did not show any significant alteration, and the morphology resulted in line with the normal myocardial structure. Similarly, $\mathrm{FtMt}^{+/+}$and untreated $\mathrm{FtMt}^{-/-}$mice showed a regular myofibrils appearance (results not shown). Only in $\mathrm{FtMt}^{-/}$mice fibril disorganization was evident, in line with the results of electron microscopy in Fig. $3 \mathrm{f}$ (results not shown).

$\mathrm{FtMt}^{--}$biochemical analyses

Since ROS formation plays a role in Dox cardiotoxicity [23, $25,37]$, we measured markers of oxidative stress in the hearts of the mice. TBARS, index of lipid peroxidation and oxidative damage, was significantly increased by Dox treatment in $\mathrm{FtMr}^{+/+}$mice, as expected, and it was also significantly higher in untreated $\mathrm{FtMt}^{-/-}$mice, in which it was further increased, but not to a significant level, by Dox treatment (Fig. 4a). Also, heme oxygenase-1 mRNA (HO-1), which is a marker of oxidative stress [38], was upregulated of about 2-fold in $\mathrm{FtMt}^{-/-}$mice, and Dox induced a small, not significant increase in HO-1 mRNA levels in both wild-type and $\mathrm{FtMt}^{-1-}$ mice (Fig. 4b). The level of oxidized proteins, as assessed by OxyBlot, was low in the hearts of wild-type animals and increased progressively with Dox and loss of FtMt, with a maximum in the $\mathrm{FtMt}^{-/}$mice exposed to Dox (Fig. 4c). We
Fig. 3 Heart morphological evaluation in mice treated with Dox. Transmission electron microphotographs of hearts from wild type mice treated with saline (a) or Dox (b), and $\mathrm{FtMt}^{-/-}$mice treated with saline (c and $\mathbf{e})$ or Dox (d and f). Arrowheads indicate damage/absence of cristae; arrows indicate fibril disarrangement. Bars $500 \mathrm{~nm}$ Three animals for each group and three sections for each sample were examined, and representative images are shown
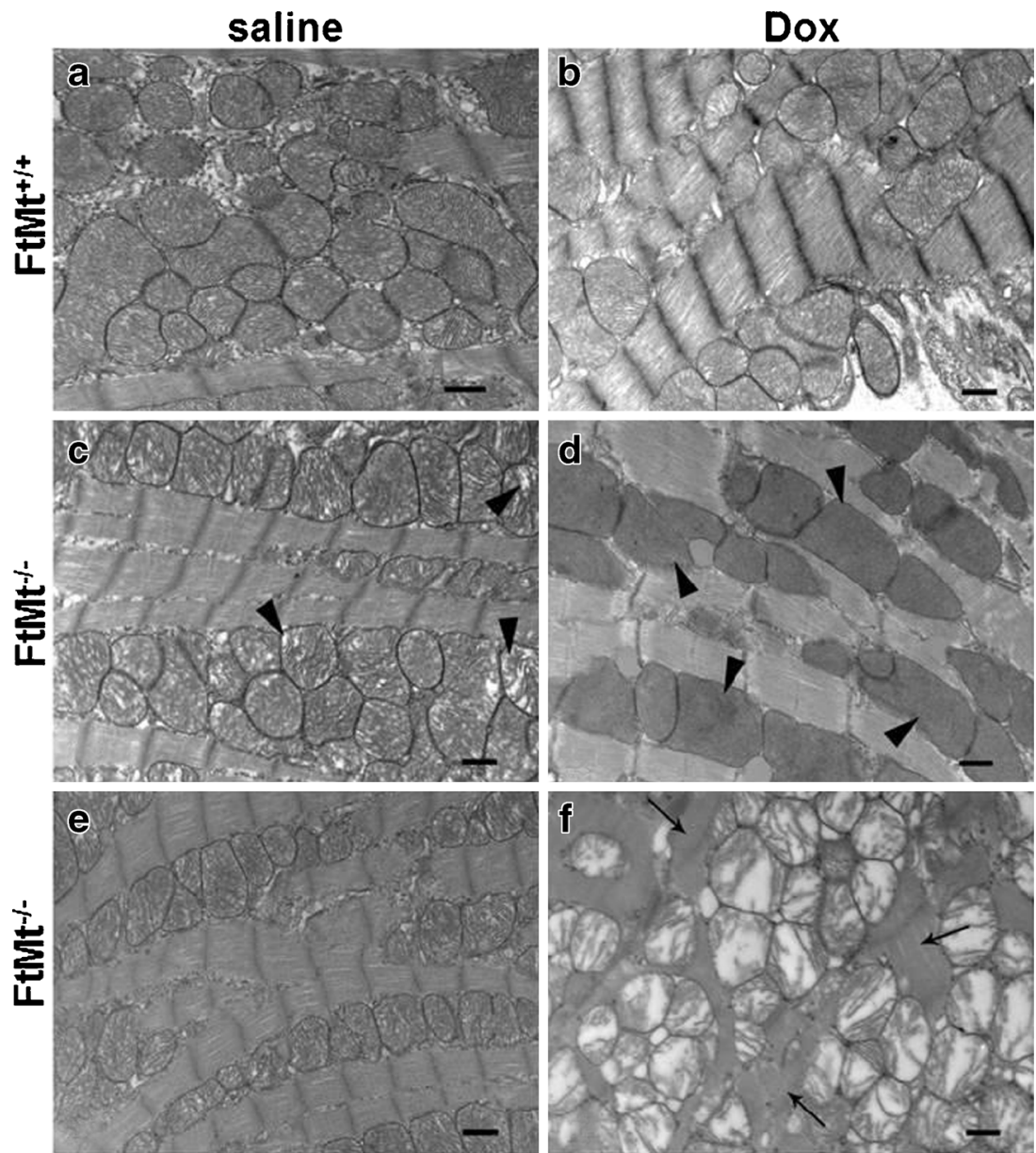
Fig. 4 Indices of oxidative stress and iron status in the heart of Dox-treated mice. Heart extracts from 8 -week-old wild-type $(+/+)$ and FtMt-deficient mice $(-/-)$ treated with Dox $15 \mathrm{mg} / \mathrm{kg}$ or saline and sacrificed after 4 days were analyzed. Oxidative stress was evaluated by measuring cardiac lipid peroxidation with the TBARS assay (a), HO-1 mRNA expression by qRT-PCR (b) and oxidized proteins by oxyblot (c). Ferritin was detected by Western blotting of non-denaturing PAGE using specific antibodies for $\mathrm{H}$ (FTH) and L ferritin (FTL); for evaluation of ferritin iron, the gels were stained with Prussian blue and then the color enhanced using DAB and $\mathrm{H}_{2} \mathrm{O}_{2}$ (Ft-iron) (d). Coomassie blue stain of the gels is shown for load calibration. At least five animals per group were analyzed, two representative samples are shown a

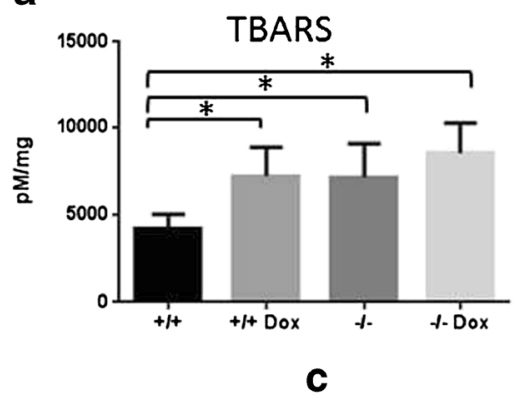

b
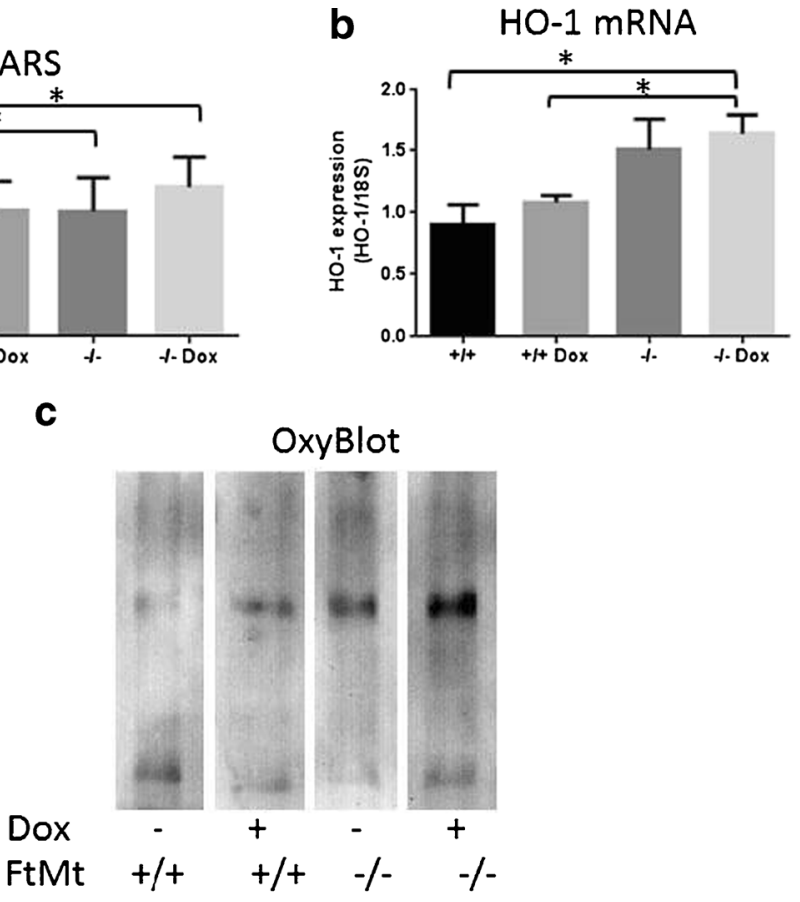

d

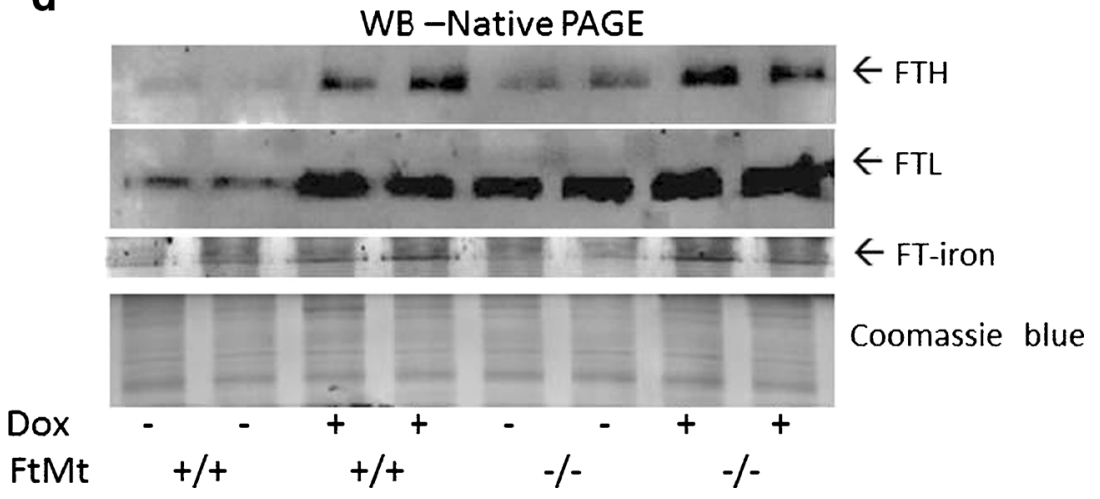

analyzed also the ferritins, since they are upregulated by Dox in $\mathrm{H} 9 \mathrm{c} 2$ cardiomyocytes and mouse heart $[36,39]$. Both $\mathrm{H}$ and $\mathrm{L}$ ferritins increased after exposure to Dox, as expected, more interesting, the basal level of both ferritins was higher in $\mathrm{FtMt}^{-/-}$than in $\mathrm{FtMt}^{+/+}$(Fig. 4d). Ferritin iron, determined by enhanced Prussian blue stain of non-denaturing PAGE, mirrored the level of ferritins (Fig. 4d).

The mitochondrial damage indicated by electron microscopy observations prompted us to evaluate ATP level as an indicator of mitochondrial functionality (Fig. 5a). Treatment with Dox resulted in a significant decrease in ATP levels compared with the controls both in wild-type and FtMtdeficient mice. No difference was found in basal levels of ATP in the two mouse lines. We also analyzed the expression of B-type natriuretic peptide (BNP), a marker of cardiac injury $[36,40]$, and we found that BNP mRNA levels were similar in both wild-type and $\mathrm{FtMt}^{-/}$mice, and were changed by Dox challenge in opposite way in the two strains, but the difference was not significant (Fig. 5b). Given that DOX is a well-known inducer of apoptosis [24], we investigated whether apoptosis was preferentially induced in $\mathrm{FtMt}^{-/-}$mice; however, in FtMtdeficient mice, caspase-3 activity was not different from that of controls and was not significantly affected by Dox treatment (Fig. 5c); moreover, p53 mRNA levels were similar in the four groups of animals (Fig. 5d). These results are in line with the lack of morphological features of apoptosis (e.g., chromatin condensation) indicated by EM (see Fig. 3).

Autophagy is a cellular process that removes damaged structures and thus provides a survival advantage to cells experiencing stress or nutrient deprivation, but excessive autophagy may cause cell death; thus, careful regulation of autophagy is important, particularly in the myocardium. Therefore, we investigated autophagic signaling by analyzing LC3, a marker of autophagosome formation that has been recently found to be induced in the skeletal muscle of rats $24 \mathrm{~h}$ after treatment with $15 \mathrm{mg} / \mathrm{kg}$ Dox [41]. Blotting of the 
Fig. 5 Indices of functionality, apoptosis, and autophagy in the heart of Dox-treated mice. Mitochondrial function and tissue damage were evaluated by measuring ATP content (a) and BNP mRNA expression (b), respectively. Apoptosis was evaluated by measuring caspase-3 activity (c) and p53 mRNA expression (d). For RT-PCR analysis of mRNA levels, samples were analyzed in triplicate and normalized to the housekeeping gene 18S RNA. Autophagy was evaluated by analyzing LC3 (e): the native (LC3-I) and lipidated (LC3-II) bands were separated on SDSPAGE and revealed by immunoblotting with anti-LC3 antibody, GAPDH was used for load calibration. The graph shows the ratio between the intensity of the lower and the upper band calculated from three independent experiments

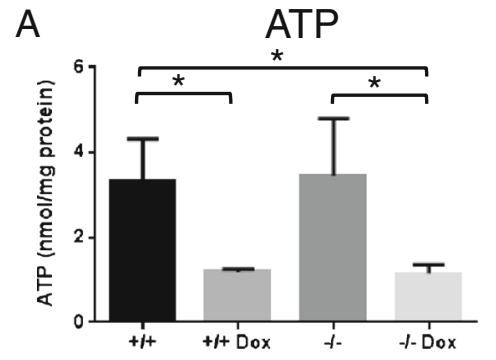

B $\quad$ BNP mRNA
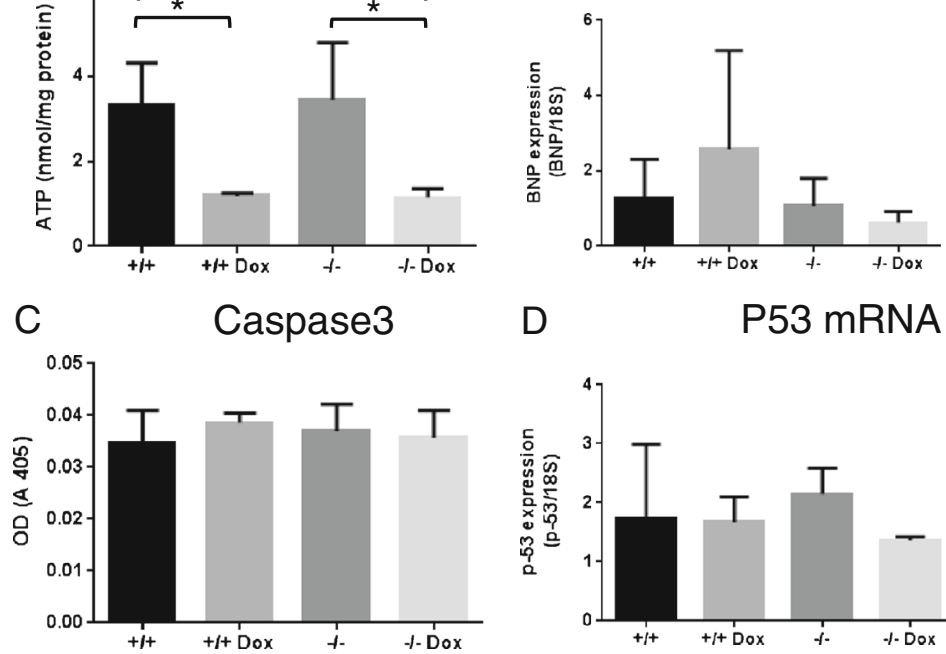

D

P53 mRNA

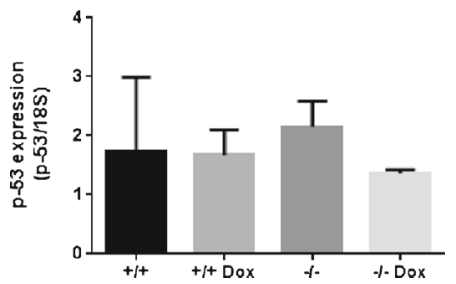

E

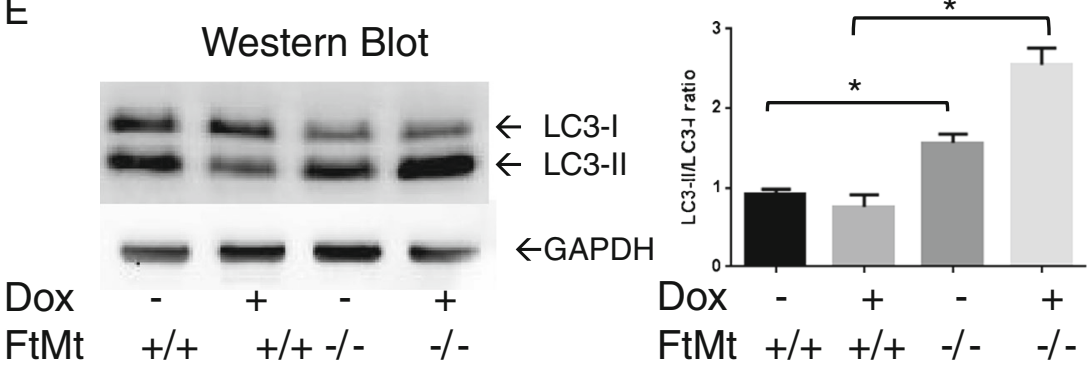

heart extracts showed a proportional increase of the lipidated LC3-II band in the $\mathrm{FtMt}^{-/-}$animals $(50 \%$ increase in LC3-II/ LC3-I ratio), compared with the $\mathrm{FtMt}^{+/+}$that was further stimulated by Dox up to $>2$-fold increase (Fig. 5e) suggesting a higher autophagy activity. Dox did not affect LC3 in the $\mathrm{FtMt}^{+/+}$mice.

Dox is known to be toxic also to testis [42]; therefore, we performed an initial morphological analysis of the testis of 8week-old mice sacrificed 30 days after Dox treatment. Histological examination of semi-thin slices showed that the testis of Dox-treated $\mathrm{FtMt}^{+/+}$had a normal morphology with tubules rich in spermatocytes, similar to those shown in Fig. 1f or g, while the morphology of testis from Dox-treated $\mathrm{FtMt}^{-1-}$ mice was largely altered with complete absence of spermatocytes (Fig. S1).

\section{Discussion}

FtMt occupies a strategic position in a site where the encounter between $\mathrm{Fe}(\mathrm{II})$ and ROS is very likely to occur and Fenton reaction to develop [43]. FtMt with its capacity to remove both $\mathrm{Fe}(\mathrm{II})$ and $\mathrm{H}_{2} \mathrm{O}_{2}$ is expected to protect the mitochondria from the development of toxic-free radicals. This hypothesis was confirmed by in vitro studies of cells that have been transfected to express FtMt at rather high levels [8]. However, FtMt is expressed only in mammals, in Drosophila [44] and also in plants [45], while it is absent in most vertebrates. Moreover, in mammals, it is expressed only by a few cell types, typically characterized by high oxidative activity. The finding that FtMt-deficient mice [22] and also FtMt-deficient Drosophila [44] are healthy and do not show any evident phenotype suggested that the functionality of FtMt is not essential under normal conditions. Moreover, a study on the DNA variations of human FtMt in patients affected by myelodysplastic syndromes and by movement disorders identified some variations, but no disabling mutations [46]. To verify the role of this gene, we produced a novel strain of FtMt-null mice, using a strategy slightly different from that used previously [22]. We used Lac-Z as a reporter gene, but we could not observe any B-gal activity in the testis of the $\mathrm{FtMt}^{-/-}$mice or in any other tissue, possibly because of the low level of expression of FtMt, or to modification of the methylation status. Our mice are healthy and iron homeostasis appears to be preserved, as described before in a different $\mathrm{FtMt}^{-/-}$strain [22].

We hypothesized that FtMt may be required under stress conditions by tissues expressing it. Heart is rich in FtMt, and FtMt protects HeLa cells from Dox [33], a well-characterized cardiotoxic agent. Moreover, Dox induces FtMt expression in 
cultured rat cardiomyocytes and causes mitochondrial iron accumulation [34]. To challenge the protective activity of FtMt in vivo in the heart, we tested a condition of acute cardiotoxicity by treating the mice with a dose of $15 \mathrm{mg} / \mathrm{kg}$ of Dox, as before [36]. Dox toxicity is strongly related to the age and gender of the mice. Thus, we restricted to 12-weekold (84 days) female mice and found that under these conditions the treatment killed all the $\mathrm{FtMt}^{-/-}$mice, but only about half of the control animals, suggesting that FtMt is protective against Dox-mediated injury.

Histological examination under light (not shown) and electron microscopy (Fig. 3a, b) did not reveal morphologic abnormalities in hearts of the Dox-treated $\mathrm{FtMt}^{+/+}$mice. However, about half of these mice were expected to die in the week after the treatment (Fig. 2). The lack of abnormalities is in agreement with published data that cardiac morphological alterations were evident after higher Dox dosages [47, 48], longer treatments [49], or repeated doses [50]. On the other hand, the $\mathrm{FtMt}^{-/-}$hearts showed mitochondrial defects even when untreated, with loss of cristae in a part of mitochondria, but the mice survived without evident health problem until 18 month of age. More important, the Dox treatment aggravated the heart abnormalities causing major alterations of mitochondrial cristae and myofibril damages in some heart portions (Fig. 3c, d). Unfortunately we could not perform direct studies on heart functionality, and thus, we could not verify experimentally that cardiac failure was the cause of the mortality of the three control mice and of the ten FtMtdeficient mice described in Fig. 2. We are aware that this is a major limitation to this study. However, we thought reasonable to infer that mice exposed to toxic doses of a cardiotoxic drug would die for heart problems. Therefore, we performed biochemical analyses on the hearts. They revealed that $\mathrm{FtMt}^{-/}$ hearts have increased lipid peroxidation, protein oxidation, and HO-1 expression, all indices of oxidative damages. We expected that the morphological alteration of the mitochondria in the FtMt-null mice was associated with a reduced functionality, but the level of ATP was very similar in the two strains. Also, the expression of cardiac BNP, an index of heart failure, was unchanged in the two strains, Moreover, we did not detect differences in indices of apoptosis, caspase-3, and p53 mRNA in the two hearts. The differences involved markers of iron status (cytosolic ferritins) and an important index of autophagy, LC3 [51]. Both increased in the FtMt-null mice. FtMt has a strong iron withdrawing capacity; thus, its absence may increase cytosolic iron and induce $\mathrm{H}$ and $\mathrm{L}$ ferritin expression, as it occurs in cultured cells [7], and also reduce the stabilization of HIF-1 and the expression of protective genes [52]. Apparently, the increase of the antioxidant activity of $\mathrm{H}$-ferritin is not sufficient to compensate the loss of that associated with FtMt. Probably more interesting was the increase of lipidated LC3. Recently, it was shown that iron chelation is a strong inducer of mitophagy in cells [53], and possibly, the local iron deregulation caused by FtMt absence might favor this process. Altogether, the data indicate that the absence of FtMt affects mitochondrial morphology, increases oxidative damage and possibly autophagy, and has an effect on cytosolic ferritins.

Dox treatment in the control mice increased oxidative damage (TBARS and Oxyblot), reduced ATP, and induced cytosolic ferritin expression, in agreement with previous studies [36]. Surprisingly, the absence of FtMt did not have a significant effect on the response to the treatment, except for higher increases in protein oxidation and LC3 induction. We found that both testes and hearts were sensitive to the Dox treatments (supplemental data), suggesting a role for FtMt in the protection against the doxorubicin-induced mitochondrial iron accumulation that was indicated responsible for cardiotoxicity [34]. In fact, mitochondrial iron accumulation occurs also in cellular models of Freidreich's ataxia where FtMt expression was protective $[9,10]$.

In conclusion, the present data indicate that the absence of FtMt alters the morphology of heart mitochondria with signs of oxidative damage. These mitochondria are more sensitive to Dox-induced damage, which results in a dramatic reduction of the mice survival, possibly due to heart failure. Notably, under our experimental conditions, heart damage 4 days after exposure to Dox is limited in wild-type mice, with no apoptosis and normal cardiac morphology. This suggests that FtMt may play a protective role under a number of not particularly severe pathophysiological conditions commonly experienced by the heart. Finally, the results of this study represent the first in vivo evidence for a function of FtMt.

Acknowledgments The authors would like to thank Ornella Azzolino for the production of the transgenic mice, Fiorella Balzac for the screening of the transfected ES cells, and Maria Gaman and Paolo Monti for the technical assistance with the electron microscopy.

This work was supported by Italian Telethon grant GGP05141 and GP10099 to PA and by a MIUR grant to GC.

Disclosure The authors declare that they have no conflict of interests.

Open Access This article is distributed under the terms of the Creative Commons Attribution License which permits any use, distribution, and reproduction in any medium, provided the original author(s) and the source are credited.

\section{References}

1. Drysdale J, Arosio P, Invernizzi R, Cazzola M, Volz A, Corsi B, Biasiotto G, Levi S (2002) Mitochondrial ferritin: a new player in iron metabolism. Blood Cells Mol Dis 29:376-383

2. Levi S, Corsi B, Bosisio M, Invernizzi R, Volz A, Sanford D, Arosio P, Drysdale J (2001) A human mitochondrial ferritin encoded by an intronless gene. J Biol Chem 276:24437-24440

3. D'estaintot B, Paolo S, Granier T, Gallois B, Chevalier J, PRECIGOUX G, Levi S, Arosio P (2004) Crystal structure and biochemical properties of the human mitochondrial ferritin and its mutant Ser144Ala. J Mol Biol 340:277-293 
4. Levi S, Arosio P (2004) Mitochondrial ferritin. Int J Biochem Cell Biol 36:1887-1889

5. Snyder AM, Wang X, Patton SM, Arosio P, Levi S, Earley CJ, Allen RP, Connor JR (2009) Mitochondrial ferritin in the substantia nigra in restless legs syndrome. J Neuropathol Exp Neurol 68:1193-1199

6. Cazzola M, Invernizzi R, Bergamaschi G, Levi S, Corsi B, Travaglino E, Rolandi V, Biasiotto G, Drysdale J, Arosio P (2003) Mitochondrial ferritin expression in erythroid cells from patients with sideroblastic anemia. Blood 101:1996-2000

7. Corsi B, Cozzi A, Arosio P, Drysdale J, Santambrogio P, Campanella A, Biasiotto G, Albertini A, Levi S (2002) Human mitochondrial ferritin expressed in HeLa cells incorporates iron and affects cellular iron metabolism. J Biol Chem 277:22430-22437

8. Campanella A, Rovelli E, Santambrogio P, Cozzi A, Taroni F, Levi S (2009) Mitochondrial ferritin limits oxidative damage regulating mitochondrial iron availability: hypothesis for a protective role in Friedreich ataxia. Hum Mol Genet 18:1-11

9. Campanella A, Isaya G, O’Neill HA, Santambrogio P, Cozzi A, Arosio P, Levi S (2004) The expression of human mitochondrial ferritin rescues respiratory function in frataxin-deficient yeast. Hum Mol Genet 13:2279-2288

10. Zanella I, Derosas M, Corrado M, Cocco E, Cavadini P, Biasiotto G, Poli M, Verardi R, Arosio P (2008) The effects of frataxin silencing in HeLa cells are rescued by the expression of human mitochondrial ferritin. Biochim Biophys Acta Mol Basis Dis 1782:90-98

11. Santambrogio P, Erba BG, Campanella A, Cozzi A, Causarano V, Cremonesi L, Gallì A, Della Porta MG, Invernizzi R, Levi S (2011) Over-expression of mitochondrial ferritin affects the JAK2/STAT5 pathway in K562 cells and causes mitochondrial iron accumulation. Haematologica 96:1424-1432

12. Invernizzi R, Travaglino E, Della Porta MG, Gallì A, Malcovati L, Rosti V, Bergamaschi G, Erba BG, Bellistri F, Bastia R et al (2013) Effects of mitochondrial ferritin overexpression in normal and sideroblastic erythroid progenitors. Br J Haematol 161:726-737

13. Tehranchi R, Invernizzi R, Grandien A, Zhivotovsky B, Fadeel B, Forsblom AM, Travaglino E, Samuelsson J, Hast R, Nilsson L et al (2005) Aberrant mitochondrial iron distribution and maturation arrest characterize early erythroid precursors in low-risk myelodysplastic syndromes. Blood 106:247-253

14. Nie G, Chen G, Sheftel AD, Pantopoulos K, Ponka P (2006) In vivo tumor growth is inhibited by cytosolic iron deprivation caused by the expression of mitochondrial ferritin. Blood 108:2428-2434

15. Nie G, Sheftel AD, Kim SF, Ponka P (2005) Overexpression of mitochondrial ferritin causes cytosolic iron depletion and changes cellular iron homeostasis. Blood 105:2161-2167

16. Santambrogio P, Biasiotto G, Sanvito F, Olivieri S, Arosio P, Levi S (2007) Mitochondrial ferritin expression in adult mouse tissues. J Histochem Cytochem 55:1129-1137

17. Jeong S, Rathore K, Schulz K, Ponka P, Arosio P, David S (2009) Dysregulation of iron homeostasis in the CNS contributes to disease progression in a mouse model of amyotrophic lateral sclerosis. J Neurosci 29:570-579

18. Shi Z, Nie G, Duan X, Rouault T, Wu W, Ning B, Zhang N, Chang Y, Zhao B (2010) Neuroprotective mechanism of mitochondrial ferritin on 6-hydroxydopamine-induced dopaminergic cell damage: implication for neuroprotection in Parkinson's disease. Antioxid Redox Signal 13:783-796

19. Lu Z, Nie G, Li Y, Soe-Lin S, Tao Y, Cao Y, Zhang Z, Liu N, Ponka P, Zhao B (2009) Overexpression of mitochondrial ferritin sensitizes cells to oxidative stress via an iron-mediated mechanism. Antioxid Redox Signal. doi:10.1089/ars.2008.2306

20. Wu WS, Zhao YS, Shi ZH, Chang SY, Nie GJ, Duan XL, Zhao SM, Wu Q, Yang ZL, Zhao BL et al (2013) Mitochondrial ferritin attenuates $\beta$-amyloid-induced neurotoxicity: reduction in oxidative damage through the Erk/P38 mitogen-activated protein kinase pathways. Antioxid Redox Signal 18:158-169
21. Yang H, Yang M, Guan H, Liu Z, Zhao S, Takeuchi S, Yanagisawa D, Tooyama I (2013) Mitochondrial ferritin in neurodegenerative diseases. Neurosci Res. doi:10.1016/j.neures.2013.07.005

22. Bartnikas TB, Campagna DR, Antiochos B, Mulhern H, Pondarré C, Fleming MD (2010) Characterization of mitochondrial ferritindeficient mice. Am J Hematol 85:958-960

23. Minotti G, Licata S, Saponiero A, Menna P, Calafiore AM, Di Giammarco G, Liberi G, Animati F, Cipollone A, Manzini S et al (2000) Anthracycline metabolism and toxicity in human myocardium: comparisons between doxorubicin, epirubicin, and a novel disaccharide analogue with a reduced level of formation and [4Fe-4S] reactivity of its secondary alcohol metabolite. Chem Res Toxicol 13: 1336-1341

24. Minotti G, Menna P, Salvatorelli E, Cairo G, Gianni L (2004) Anthracyclines: molecular advances and pharmacologic developments in antitumor activity and cardiotoxicity. Pharmacol Rev 56: $185-229$

25. Chen B, Peng X, Pentassuglia L, Lim CC, Sawyer DB (2007) Molecular and cellular mechanisms of anthracycline cardiotoxicity. Cardiovasc Toxicol 7:114-121

26. Goormaghtigh E, Huart P, Praet M, Brasseur R, Ruysschaert JM (1990) Structure of the adriamycin-cardiolipin complex. Role in mitochondrial toxicity. Biophys Chem 35:247-257

27. Salvatorelli E, Guarnieri S, Menna P, Liberi G, Calafiore AM, Mariggiò MA, Mordente A, Gianni L, Minotti G (2006) Defective one- or two-electron reduction of the anticancer anthracycline epirubicin in human heart. Relative importance of vesicular sequestration and impaired efficiency of electron addition. J Biol Chem 281: 10990-11001

28. Octavia Y, Tocchetti CG, Gabrielson KL, Janssens S, Crijns HJ, Moens AL (2012) Doxorubicin-induced cardiomyopathy: from molecular mechanisms to therapeutic strategies. J Mol Cell Cardiol 52: $1213-1225$

29. Link G, Tirosh R, Pinson A, Hershko C (1996) Role of iron in the potentiation of anthracycline cardiotoxicity: identification of heart cell mitochondria as a major site of iron-anthracycline interaction. $\mathrm{J}$ Lab Clin Med 127:272-278

30. Hershko C, Link G, Tzahor M, Kaltwasser JP, Athias P, Grynberg A, Pinson A (1993) Anthracycline toxicity is potentiated by iron and inhibited by deferoxamine: studies in rat heart cells in culture. $\mathrm{J} \mathrm{Lab}$ Clin Med 122:245-251

31. Miranda CJ, Makui H, Soares RJ, Bilodeau M, Mui J, Vali H, Bertrand R, Andrews NC, Santos MM (2003) Hfe deficiency increases susceptibility to cardiotoxicity and exacerbates changes in iron metabolism induced by doxorubicin. Blood 102:2574-2580

32. Panjrath GS, Patel V, Valdiviezo CI, Narula N, Narula J, Jain D (2007) Potentiation of Doxorubicin cardiotoxicity by iron loading in a rodent model. J Am Coll Cardiol 49:2457-2464

33. Cocco E, Porrini V, Derosas M, Nardi V, Biasiotto G, Maccarinelli F, Zanella I (2013) Protective effect of mitochondrial ferritin on cytosolic iron dysregulation induced by doxorubicin in HeLa cells. Mol Biol Rep 40:6757-6764

34. Ichikawa Y, Ghanefar M, Bayeva M, Wu R, Khechaduri A, Naga Prasad SV, Mutharasan RK, Naik TJ, Ardehali H (2014) Cardiotoxicity of doxorubicin is mediated through mitochondrial iron accumulation. J Clin Invest 124:617-630

35. Liu P, Jenkins NA, Copeland NG (2003) A highly efficient recombineering-based method for generating conditional knockout mutations. Genome Res 13:476-484

36. Corna G, Galy B, Hentze MW, Cairo G (2006) IRP1-independent alterations of cardiac iron metabolism in doxorubicin-treated mice. $\mathrm{J}$ Mol Med 84:551-560

37. Stěrba M, Popelová $\mathrm{O}$, Vávrová $\mathrm{A}$, Jirkovský E, Kovaříková $\mathrm{P}$, Geršl V, Simůnek T (2013) Oxidative stress, redox signaling, and metal chelation in anthracycline cardiotoxicity and pharmacological cardioprotection. Antioxid Redox Signal 18:899-929 
38. Applegate LA, Luscher P, Tyrrell RM (1991) Induction of heme oxygenase: a general response to oxidant stress in cultured mammalian cells. Cancer Res 51:974-978

39. Corna G, Santambrogio P, Minotti G, Cairo G (2004) Doxorubicin paradoxically protects cardiomyocytes against iron-mediated toxicity: role of reactive oxygen species and ferritin. J Biol Chem 279: 13738-13745

40. Kuhn M, Voss M, Mitko D, Stypmann J, Schmid C, Kawaguchi N, Grabellus F, Baba HA (2004) Left ventricular assist device support reverses altered cardiac expression and function of natriuretic peptides and receptors in end-stage heart failure. Cardiovasc Res 64:308314

41. Smuder AJ, Kavazis AN, Min K (1985) Powers SK (2011) Exercise protects against doxorubicin-induced markers of autophagy signaling in skeletal muscle. J Appl Physiol 111:1190-1198

42. Takahashi H, Tainaka H, Umezawa M, Takeda K, Tanaka H, Nishimune Y, Oshio S (2011) Evaluation of testicular toxicology of doxorubicin based on microarray analysis of testicular specific gene expression. J Toxicol Sci 36:559-567

43. Figueira TR, Barros MH, Camargo AA, Castilho RF, Ferreira JC, Kowaltowski AJ, Sluse FE, Souza-Pinto NC, Vercesi AE (2013) Mitochondria as a source of reactive oxygen and nitrogen species: from molecular mechanisms to human health. Antioxid Redox Signal 18:2029-2074

44. Missirlis F, Holmberg S, Georgieva T, Dunkov BC, Rouault TA, Law JH (2006) Characterization of mitochondrial ferritin in Drosophila. Proc Natl Acad Sci U S A 103:5893-5898

45. Zancani M, Peresson C, Biroccio A, Federici G, Urbani A, Murgia I, Soave C, Micali F, Vianello A, Macri F (2004) Evidence for the presence of ferritin in plant mitochondria. Eur J Biochem 271:36573664

46. Castiglioni E, Finazzi D, Goldwurm S, Levi S, Pezzoli G, Garavaglia B, Nardocci N, Malcovati L, Porta M, Gallì A et al (2010) Sequence variations in mitochondrial ferritin: distribution in healthy controls and different types of patients. Genet Test Mol Biomarkers, Ferritin. doi:10.1089/gtmb.2010.0076

47. Kang YJ, Chen Y, Yu A, Voss-McCowan M, Epstein PN (1997) Overexpression of metallothionein in the heart of transgenic mice suppresses doxorubicin cardiotoxicity. J Clin Invest 100:1501-1506

48. Li K, Sung RY, Huang WZ, Yang M, Pong NH, Lee SM, Chan WY, Zhao H, To MY, Fok TF et al (2006) Thrombopoietin protects against in vitro and in vivo cardiotoxicity induced by doxorubicin. Circulation 113:2211-2220

49. Suliman HB, Carraway MS, Ali AS, Reynolds CM, Welty-Wolf KE, Piantadosi CA (2007) The CO/HO system reverses inhibition of mitochondrial biogenesis and prevents murine doxorubicin cardiomyopathy. J Clin Invest 117:3730-3741

50. Sun X, Zhou Z, Kang YJ (2001) Attenuation of doxorubicin chronic toxicity in metallothionein-overexpressing transgenic mouse heart. Cancer Res 61:3382-3387

51. Romao S, Münz C (2014) LC3-associated phagocytosis. Autophagy 10

52. Spagnuolo RD, Recalcati S, Tacchini L, Cairo G (2011) Role of hypoxia-inducible factors in the dexrazoxane-mediated protection of cardiomyocytes from doxorubicin-induced toxicity. $\mathrm{Br} \mathrm{J}$ Pharmacol 163:299-312

53. Allen GF, Toth R, James J, Ganley IG (2013) Loss of iron triggers PINK1/Parkin-independent mitophagy. EMBO Rep 14:1127-1135 